\title{
Preliminary space mission design under uncertainty
}

\author{
Nicolas Croisard ${ }^{\mathrm{a}, *}$, Massimiliano Vasile ${ }^{\mathrm{a}}$, Stephen Kemble ${ }^{\mathrm{b}}$, Gianmarco Radice ${ }^{\mathrm{a}}$ \\ a University of Glasgow, Glasgow, UK \\ ${ }^{\mathrm{b}}$ Astrium Ltd., Hertfordshire, UK
}

\section{A R T I C L E I N F O}

\section{Article history:}

Received 28 January 2009

Received in revised form

10 August 2009

Accepted 11 August 2009

\section{Keywords:}

Evidence Theory

Optimisation under uncertainties

Preliminary design

\begin{abstract}
A B S T R A C T
This paper proposes a way to model uncertainties and to introduce them explicitly in the design process of a preliminary space mission. Traditionally, a system margin approach is used in order to take them into account. In this paper, Evidence Theory is proposed to crystallise the inherent uncertainties. The design process is then formulated as an optimisation under uncertainties (OUU). Three techniques are proposed to solve the OUU problem: (a) an evolutionary multi-objective approach, (b) a step technique consisting of maximising the belief for different levels of performance, and (c) a clustering method that firstly identifies feasible regions. The three methods are applied to the BepiColombo mission and their effectiveness at solving the OUU problem are compared.
\end{abstract}

(c) 2009 Elsevier Ltd. All rights reserved.

\section{Introduction}

In the early phase of the design of a space mission, it is generally desirable to investigate as many feasible alternative solutions as possible. At this particular stage, an insufficient consideration for uncertainty would lead to a wrong decision on the feasibility of the mission. Traditionally, a system margin approach is used in order to take into account the inherent uncertainties related to the computation of the system budgets. The reliability of the mission is then independently computed in parallel. An iterative, though integrated, process between the solution design and the reliability assessment should finally converge to an acceptable solution.

This paper proposes a way to model uncertainties with Evidence Theory (ET) and to introduce them explicitly in the design process. The overall system design is then optimised, minimising the impact of uncertainties on the optimal value of the design criteria (e.g. minimum system mass, minimum system power, etc.). The minimisation of the impact of

\footnotetext{
* Corresponding author.

E-mail addresses: n.croisard@aero.gla.ac.uk (N. Croisard), mvasile@aero.gla.ac.uk (M. Vasile), stephen.kemble@astrium.eads.net (S. Kemble), gradice@aero.gla.ac.uk (G. Radice).
}

uncertainties in the design process is generally known as robust design and the associated optimisation process robust optimisation. In the last few decades, robust design has been gaining wide attention and its applications have been extended from improving the quality of individual components to the design of complex engineering systems. The methods for robust design have progressed from the initial Taguchi's "parameter design method" (Taguchi, 1950) to recent nonlinear programming methods that formulate robust design problems as nonlinear optimisation problems with multiple objectives subject to feasibility robustness.

With Evidence Theory, also know as Dempster-Shafer's theory [1,2], both aleatory and epistemic uncertainties, coming from a poor or incomplete knowledge of the design parameters, can be correctly modelled. The values of uncertain or vague design parameters are expressed by means of intervals with associated probability. Each expert participating in the design, assigns an interval and a probability according to their experience. Ultimately, all the pieces of information associated to each interval are fused together to yield two cumulative values, Belief and Plausibility, that express the confidence range in the optimal design point. In particular the value of Belief expresses the lower probability that the selected design point remains optimal (and feasible) even under uncertainties. 
The use of Evidence Theory for robust engineering design was proposed in 2002 by Oberkampf et al. [3] and was more recently applied to the robust design of space systems and space trajectories [4,5]. In this paper, Evidence Theory is applied to the optimal design of the mission BepiColombo. The main spacecraft subsystems are modelled using Evidence Theory to deal with uncertain parameters. The design process is then formulated as an optimisation under uncertainties (OUU) problem and the Belief is optimised (maximised) together with all the other criteria that define the optimality of the design point.

Note that the OUU is more computationally expensive than an optimisation without uncertainty and of the classical margin approach. However, the benefits of robust design optimisation, clearly presented in [17], are considerable. Compared to standard optimisation, it provides solutions that are poorly affected by the uncertainty in the design parameter and gives a rigorous quantitative measure of the impact of the uncertainty on the design budgets. This later aspect is a key advantage with respect to the margin approach. A demonstration of the actual ability of robust design optimisation (and of Evidence Theory) to produce better designs compared to the margin approach, in a real scenario, is out of the scopes of this paper. The main objective, here, is to present some computational techniques to deal with the OUU when Evidence Theory is used to model uncertainty. The case study presented in this paper will illustrate the use of Evidence Theory in the robust design of a space mission.

Three techniques are proposed to solve the related OUU problem: (a) an evolutionary multi-objective approach aiming at minimising the effect of uncertainties on the objective function, while optimising the mission goals, (b) a step technique consisting of maximising the belief for different levels of performance using a local optimiser, and (c) a clustering method that identifies, in the space of design and uncertain parameters, the set of points for which the design criteria assume values below a given threshold.

The results in the paper show the effectiveness of the three proposed techniques at solving efficiently the OUU problem.

\section{Uncertainty modelling through Evidence Theory}

The most common way to deal with uncertainty is the probabilistic approach [18]. However, this theory does not suit to represent every type of uncertainties, and unavoidable assumptions may significantly modify the result of the analysis. Thus new theories have been developed. Evidence Theory is one of them and is currently the most common alternative to probability $[3,19]$.

In this section, the different types of uncertainties are described, leading to the justification of why probability theory may not be suitable in our application. Finally, Evidence Theory is presented and proposed as an alternative.

\subsection{Types of uncertainty}

Uncertainties are usually classified in two distinct categories, aleatory and epistemic uncertainty. According to Helton [6], the definition of each type is ${ }^{1}$ :

${ }^{1}$ This comes actually from [7], citing Helton.
Aleatory uncertainty: The type of uncertainty which results from the fact that a system can behave in random ways.

Epistemic uncertainty: The type of uncertainty which results from the lack of knowledge about a system and is a property of the analysts performing the analysis.

W.L. Oberkampf considers a third category, Error, also called numerical uncertainty, which "is defined as a recognizable deficiency in any phase or activity of modelling and simulation that is not due to lack of knowledge" [8]. Such uncertainties are well-known, and a good estimation of the error should be easily available. This point distinguishes errors to epistemic uncertainties.

Aleatory uncertainties are due to the random nature of input data while epistemic ones are generally linked to incomplete modelling of the physical system, the boundary conditions, unexpected failure modes, etc. In the particular case of preliminary space mission design, analysts face both types of uncertainty. For example, the initial velocity of the spacecraft, the gravity model or the solar radiation presents aleatory uncertainties. However, most of the parameters of the spacecraft subsystems are first assessed by a group of experts, expressing their opinion on ranges of values. The uncertainty associated to those parameters is therefore epistemic.

The classical way to treat uncertainty, through probability theory, requires an assumption on the probability density function associated to a phenomenon. A probability density function is well suited to mathematically model aleatory uncertainties, as far as enough data (experimental for instance) are available [8]. Even though, the analyst still has to assume the distribution function and estimate its parameters. Moreover, Bae et al. [9] pointed out that aleatory uncertainty could be in fact epistemic uncertainty when "insufficient data are available to construct a probability distribution".

Probability fails to represent epistemic uncertainties because there is no reason to prefer one distribution function over another [3]. When uncertainties are expressed by means of intervals, based on experts' opinion or rare experimental data, as it is the case in space mission design, this representation becomes even more questionable.

A few modern theories exist [17] to better represent epistemic uncertainties, without the need to make additional assumptions. The most common one is Evidence Theory, which we propose to use in the framework of preliminary space mission design.

\subsection{Overview of Evidence Theory}

Evidence Theory was developed by Shafer [2] based on Dempster's original work [1] and has been proven to model adequately both types of uncertainty. The theory does not request additional assumptions when the available information is poor or incomplete. For instance, evidence on the event $A$ or $B$ does not imply/require information on both events $A$ and $B$. Similarly, the knowledge of an event does not imply knowledge of its opposite (for the probability theory, $P(\bar{A})=1-P(A))$. Moreover, it is common to have the information from diverse sources, such as different experts or experiments. There is no reason, a priori, to choose one source from the others. Evidence Theory offers the possibility 
to combine information from different sources. A number of rules have been developed to combine evidence depending on the context or how much they conflict. For a complete list of rules, the interested reader can refer to the work of Sentz and Ferson [7].

\subsubsection{Frame of discernment $\mathscr{U}$ and basic probability assignment}

The frame of discernment $\mathscr{U}$, also known as the universal set, is "a set of mutually exclusive elementary propositions" [9]. In most engineering applications of the Evidence Theory, experts express their belief of an uncertain parameter $u$ being within various intervals. $u \in[a, b]$ is then in this case an elementary proposition, thus an element of $\mathscr{U}$. The level of confidence an expert has on an elementary proposition is quantified using the Basic Probability Assignment (BPA) that satisfies the three following axioms:

1. $m(E) \geq 0, \forall E \in \mathscr{U}$,

2. $m(\varnothing)=0$, and

3. $\sum_{E \in \mathscr{U}} m(E)=1$.

An element of $\mathscr{U}$ that has a non-zero BPA is named a focal element $(F E)$.

When more than one parameter are considered uncertain (e.g. $u_{1}$ and $u_{2}$ ), the frame of discernment is composed of all the cartesian products of the intervals. The BPA of a given cartesian product is then the product of the BPA of each interval:

$$
\begin{aligned}
& m\left(\left(u_{1}, u_{2}\right) \in\left[a_{1}, b_{1}\right] \times\left[a_{2}, b_{2}\right]\right) \\
& \quad=m\left(u_{1} \in\left[a_{1}, b_{1}\right]\right) * m\left(u_{2} \in\left[a_{2}, b_{2}\right]\right)
\end{aligned}
$$

\subsubsection{Belief and plausibility functions}

As stated above, Probability Theory requires an assumption on the probability distribution associated to a phenomenon in order to compute the probability of an event to happen. Such a probability is the uncertainty quantification associated to that event. However, the knowledge of the probability distribution is an important piece of information that is not always available. Evidence Theory makes use only of the available information and produces two quantities, the Belief (Bel) and the Plausibility ( $P l)$ functions, that represent the lower and upper bounds on the uncertainty quantification. They are defined as follows:

$$
\begin{aligned}
& \operatorname{Bel}(\mathbf{A})=\sum_{\substack{F E \subset \mathbf{A} \\
F E \in \mathscr{U}}} m(F E) \\
& \operatorname{Pl}(\mathbf{A})=\sum_{\substack{F E \cap \mathbf{A} \neq \varnothing \\
F E \in \mathscr{U}}} m(F E)
\end{aligned}
$$

Thus, propositions intercepting the set $\mathbf{A}$ but not included in A are considered in $P l$ but not in Bel. Let us have a look at an example. Fig. 1 represents a BPA structure of two uncertain parameters $u_{1}$ and $u_{2}$. Parameter $u_{1}$ can take its value within the four intervals $\left[a_{1}, b_{1}\right],\left[b_{1}, c_{1}\right],\left[c_{1}, d_{1}\right]$ and $\left[d_{1}, e_{1}\right]$ while the domain of $u_{2}$ is divided in three parts $\left[a_{2}, b_{2}\right]$, $\left[b_{2}, c_{2}\right]$ and $\left[c_{2}, d_{2}\right]$. Thus there is a total of twelve focal elements $F E_{1}, \ldots, F E_{12}$. Let us define the proposition $\mathbf{A}$ as the

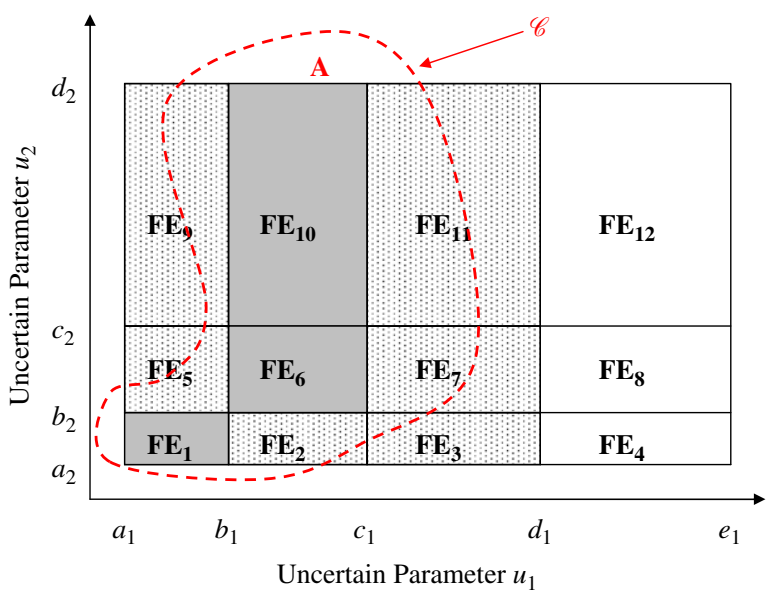

Fig. 1. Belief and plausibility of proposition $A$ in a given $B P A$ structure of two uncertain parameters.

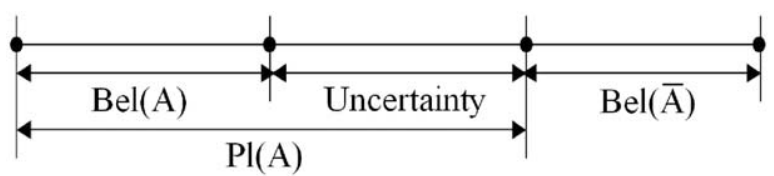

Fig. 2. Interpretation of the relation between belief, plausibility and uncertainty.

area within the dash curve $\mathscr{C}$. Only the focal elements $F E_{1}$, $F E_{6}$ and $F E_{10}$ (grey in the figure) are entirely surrounded by $\mathscr{C}$. In addition, $F E_{2}, F E_{3}, F E_{5}, F E_{7}, F E_{9}$ and $F E_{11}$ are partly inside $\mathscr{C}$ (doted in the figure), therefore only partially implying the proposition $\mathbf{A}$. Therefore the belief and plausibility of $\mathbf{A}$ are

$$
\begin{aligned}
\operatorname{Bel}(\mathbf{A}) & =m\left(F E_{1}\right)+m\left(F E_{6}\right)+m\left(F E_{10}\right) \\
P l(\mathbf{A}) & =m\left(F E_{1}\right)+m\left(F E_{2}\right)+m\left(F E_{3}\right)+m\left(F E_{5}\right)+m\left(F E_{6}\right)+m\left(F E_{7}\right) \\
& +m\left(F E_{9}\right)+m\left(F E_{10}\right)+m\left(F E_{11}\right)
\end{aligned}
$$

If the pair $\left(u_{1}, u_{2}\right)$ takes its value within $\left[b_{1}, c_{1}\right] \times\left[c_{2}, d_{2}\right]$, it fulfils proposition A. However, if it is inside $\left[c_{1}, d_{1}\right] \times\left[b_{2}, c_{2}\right]$, it may verify $\mathbf{A}$ in some cases but not always. Therefore, the belief represents our confidence in $\mathbf{A}$ to be true while the plausibility our confidence in $\mathbf{A}$ to be possible.

An important and meaningful relation between belief and plausibility functions comes directly from the fact that all basic assignments must sum to 1 .

$P l(\mathbf{A})+\operatorname{Bel}(\overline{\mathbf{A}})=1$

where $(\overline{\mathbf{A}})$ represents the complement of $\mathbf{A}$. This means that $\mathrm{Pl}$ considers the uncertainty, while Bel does not (cf. Fig. 2).

\subsection{Generic problem of optimisation under uncertainty}

Let us consider a function $f: \mathbb{R}^{m+n} \rightarrow \mathbb{R}$ characterising a system to be optimised. The function $f$ represents the system budgets (e.g. delta $V$ budget, power budget, mass budget, etc.), in the case of a space mission, and depends on some 
uncertain parameters $\mathbf{u}=\left[u_{1}, u_{2}, \ldots, u_{m}\right] \in \mathscr{U} \subseteq \mathbb{R}^{m}$ and design variables $\mathbf{d}=\left[d_{1}, d_{2}, \ldots, d_{n}\right] \in \mathscr{D} \subseteq \mathbb{R}^{n}$.

A BPA is associated to the frame of discernment $\mathscr{U}$ of the uncertain parameters $\mathbf{u}$. The design variables are well know and can be adjusted at will by the designer within a domain $\mathscr{D}$ to optimise the system. For a given constant $v$, named the threshold, the generic problem of optimisation under uncertainty (OUU) can be defined as follows:

$\max _{\mathbf{d} \in \mathscr{D}} \operatorname{Bel}(f(\mathbf{d}, \mathbf{u})<v)$

Note that, the plausibility could be used instead of the belief, or the proposition could be replaced by $f>v$, depending on the system and goal of the optimisation. The computational techniques in this paper would be equally applicable in all cases.

To better understand how the belief of $f<v$ is computed, let us define, for a given design vector $\mathbf{d}$ :

$$
\begin{aligned}
\mathscr{U}_{v} & =\{F E \in \mathscr{U} \mid \forall \mathbf{u} \in F E, f(\mathbf{d}, \mathbf{u})<v\} \\
& =\left\{F E \in \mathscr{U} \mid \max _{\mathbf{u} \in F E}(f(\mathbf{d}, \mathbf{u}))<v\right\}
\end{aligned}
$$

Thus using Eq. (2) we have

$$
\operatorname{Bel}_{\mathscr{U}}(f(\mathbf{d}, \mathbf{u})<v)=\sum_{F E \in \mathscr{U}_{v}} m(F E)
$$

From the above definitions, it can be deduced that the computational time required to solve the OUU problem can become quickly prohibitive. In fact, in order to identify $\mathscr{U}_{v}$, the maximum of $f$ over every focal element of $\mathscr{U}$ must be computed and compared to $v$. In the event that the system function is convex, this maximum lies at one of the vertices of the focal element. Otherwise, an optimisation problem has to be solved for every focal element and for each new design vector. Furthermore, because of the cartesian product in (1) the number of focal elements increases exponentially with the number of uncertain parameters and associated intervals.

Finally, designers are usually interested in the variation of the optimal belief with the threshold. Indeed, it may be relevant to take a little more risk (a slightly lower value of the belief) if the performance gain is significant. Therefore, in practise the designers are interested in the trade-off curve, solution of the bi-objective optimisation problem:

$$
\begin{cases}\max _{v \in \mathbb{R}, \mathbf{d} \in \mathscr{D}} & \operatorname{Bel}(f(\mathbf{d}, \mathbf{u})<v) \\ \min _{v \in \mathbb{R}, \mathbf{d} \in \mathscr{D}} & v\end{cases}
$$

The typical shape of the Pareto front of problem (7) is given in Fig. 3. The complexity associated to the computation of Belief and Plausibility together with the requirement to solve bi-objective problem (7) demand for an appropriate computational approach that can make Evidence Theory an interesting tool for robust design optimisation of a space mission. In the remainder of the paper some techniques will be presented and discussed.

\section{Three approaches to solve the OUU problem}

The cost of the computation of the belief function and its step-wise structure makes its maximisation difficult.

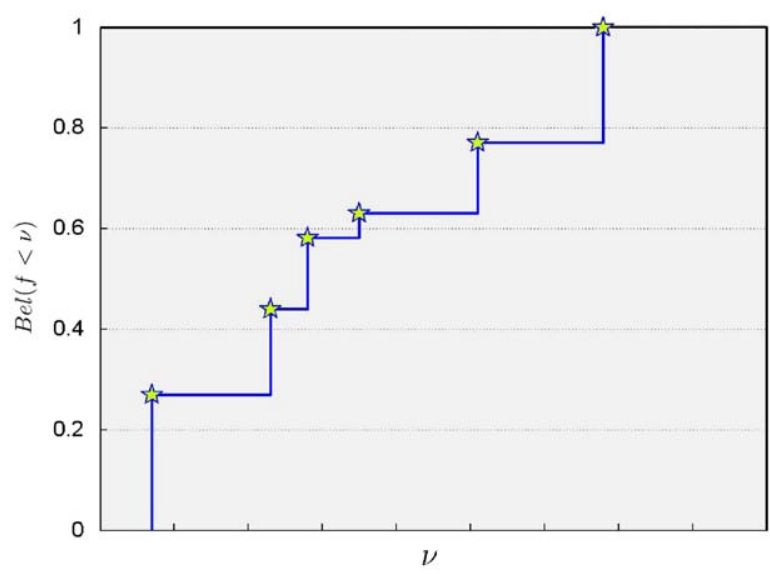

Fig. 3. Typical shape of the Pareto front of the optimisation under uncertainty problem. The stars represent the Pareto optimal points. (This is also representative of the shape of the belief function for a specific design vector.)

In this section three different approaches to solve the problem defined in Eq. (7) are presented.

\subsection{Direct approach: using a multi-objective optimiser}

The most natural way to solve problem (7) is to use a multi-objective optimiser (MOO) [4,5]. It allows investigating globally the design space and provides optimal designs for various levels of the belief. This is desirable as it would enable the decision makers to do a trade-off among them.

The direct application of a MOO algorithm to problem (7) requires the definition of a dominance index quantifying the Pareto optimality of a given design point. Let us introduce the standard dominance index:

$I_{i}=\left|\left\{j \mid \operatorname{Bel}\left(\mathbf{d}_{j}\right)>\operatorname{Bel}\left(\mathbf{d}_{i}\right) \wedge v_{j}<v_{i}, \quad j=1, \ldots, n_{p o p} \wedge j \neq i\right\}\right|$

where $|$.$| denotes the cardinality of a set and n_{p o p}$ is the number of available design vectors. For the tests in this paper we will use a population-based optimiser called EPIC with the standard formulation of the dominance index (8). EPIC is based on an algorithm that combines a deterministic domain decomposition technique and a stochastic-based multi-agent collaborative search. The interested reader can find more details in [10-12].

The problem with dominance index (8) is that each design vector corresponds to a full Bel-v curve, i.e. a set of points in the image space, one for each values of $v$ (the stars of Fig. 3), and not a single one. Moreover, the same Pareto front could correspond to different design points. As we will see in the remainder of this paper, these two difficulties can lead to a significant overhead in the computational effort required to a MOO algorithm to identify the global Pareto front.

In order to address some of the problems related to the direct application of an MOO algorithm to the solution of (7) we propose two other alternative approaches to the problem. One treats $v$ separately, maximising Bel for given values of $v$, the other identifies at first the subsets in $\mathscr{U} \times \mathscr{D}$ that 
satisfy the condition $f<v$ and then computes the value of the Bel function.

\subsection{Step method}

To be able to compute the Belief, a threshold has to be set in some way. The Step Method is very straightforward as it computes the optimum belief for discrete values of the threshold. An initial value is chosen by the user such that a design vector $\mathbf{d}$ exists for which the Belief is equal to 1 . Then the threshold is decreased (or increased, depending on the problem) and a local optimiser is used to find a optimal d starting from the previous one. This process stops when the belief reaches the minimum possible value, i.e. 0 . This procedure is detailed in Algorithm 1.

Algorithm 1. Step method.

Input: $v$, step

Output: Matrix Out where each row corresponds to a step. The $i$ th row of Out is composed of the value of the threshold, the optimum design vector and the maximum belief found at the $i$ th step.

/* This first while loop should be avoided by */ setting a high enough threshold

while $\nexists \mathbf{d} \in \mathscr{D} \mid \mathrm{Bel}^{*}(v)=1$ do

$v \leftarrow v+$ step

endw

$B e l_{\text {max }} \leftarrow 1$

while $B e l_{\max }>0$ do

$I^{*}$ Update the threshold

$v \leftarrow v-$ step

$l^{*}$ Optimisation of the belief for the given */ threshold

$\left[\right.$ Bel $\left._{\text {opt }}, \mathbf{d}_{\text {opt }}\right] \leftarrow \max _{\mathbf{d} \in \mathscr{D}} \operatorname{Bel}(f(\mathbf{d}, \mathbf{u})<v)$

$I^{*}$ Add a line at the end of the output matrix */ and save the results

Out $($ end $+1,:)=\left[v\right.$, Bel $\left._{\text {opt }}, \mathbf{d}_{\text {opt }}\right]$

$I^{*}$ Update the optimum belief variable

$\mathrm{Bel}_{\text {max }} \leftarrow B e l_{\text {opt }}$

endw

Due to the non-derivative nature of the belief function, a gradient-based optimiser is not applicable. Therefore, a derivative-free algorithm (the MatLab fminsearch algorithm) was used. A drawback of the step method is that it can fail to converge to the global Pareto front or to identify multiple design points. In fact, the use of the previous optimal d to start a new step helps the local optimiser to converge quickly but prevents the identification of a completely different design point. In order to overcome this difficulty, a multi-start approach could be used, or even a global optimiser operating on $v$ only. However, the associated extra computational time would be significant.

\subsection{Cluster approximation}

The cluster approximation is an indirect way to solve the problem described in Eq. (7). The main idea is to identify within the complete domain (i.e. the product of the

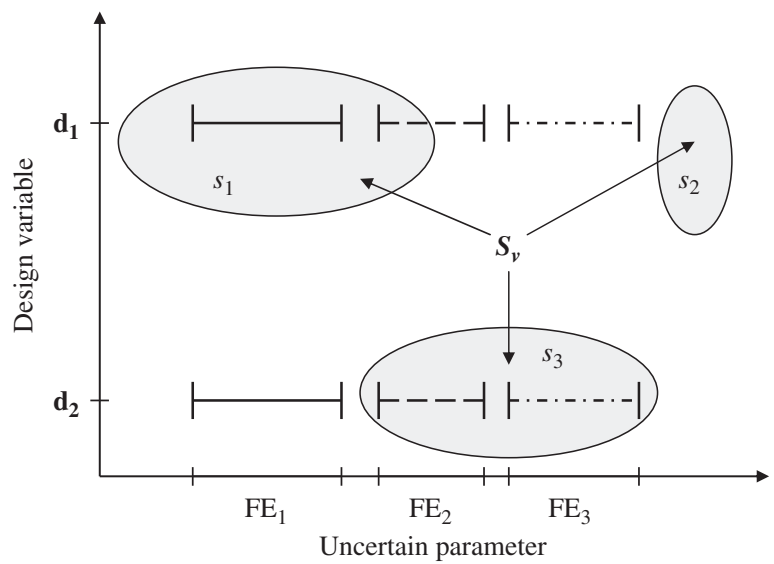

Fig. 4. Illustration of the cluster method with three focal elements $F E_{1}$, $F E_{2}$ and $F E_{3}$. The proposition $f<v$ is true only within the subdomains $s_{1}$, $s_{2}$ and $s_{3}$. Two examples of design point $\mathbf{d}_{1}$ and $\mathbf{d}_{2}$ are given.

uncertain parameters domain and the design domain) the set $\mathscr{S}_{v}$ where the system function verifies the proposition $f<v$. An approximated $\tilde{B e l}$ (resp. plausibility $\tilde{P l}$ ) of the proposition $f<v$ can then be cheaply computed by adding the mass of the focal elements included (resp. intersecting) any element of $\mathscr{S}_{v}$.

$$
\begin{aligned}
& \tilde{\operatorname{Bel}}(f<v)=\sum_{\substack{F E \subset S \\
s \in \mathscr{S}_{v}}} m(F E) \\
& \tilde{P} l(f<v)=\sum_{\substack{F E \cap \mathcal{T}_{0} \neq 0 \\
s \in \mathscr{S}_{v}}} m(F E)
\end{aligned}
$$

Fig. 4 illustrates the proposed method. In this example, there are only three focal elements $F E_{1}, F E_{2}$ and $F E_{3}$. The set of subdomains where the system function verifies the proposition is

$\mathscr{S}_{v}=\left\{s_{1}, s_{2}, s_{3}\right\}$

Two different design points $\mathbf{d}_{1}$ and $\mathbf{d}_{2}$ are represented. For $\mathbf{d}_{1}$, the approximations of the belief and plausibility are

$$
\begin{aligned}
& \tilde{B e l}_{\mathbf{d}_{1}}(f<v)=m\left(F E_{1}\right) \\
& \tilde{P} l_{\mathbf{d}_{1}}(f<v)=m\left(F E_{1}\right)+m\left(F E_{2}\right)
\end{aligned}
$$

For $\mathbf{d}_{2}$, the approximations of the belief and plausibility are

$$
\begin{aligned}
& \tilde{B e l}_{\mathbf{d}_{2}}(f<v)=m\left(F E_{2}\right)+m\left(F E_{3}\right) \\
& \tilde{P} l_{\mathbf{d}_{2}}(f<v)=m\left(F E_{2}\right)+m\left(F E_{3}\right)
\end{aligned}
$$

To compute the approximation of the Bel function, the set $\mathscr{S}_{v}$ is computed for increasing values of the threshold until a belief of 1 is found. At each step, sample points verifying the proposition $f(\mathbf{d}, \mathbf{u})<v$ are identified, then classified in clusters. The points of a given cluster define one subset $s_{i}$ of $\mathscr{S}_{v}$. Then, the design maximising the approximation of the belief Bel is selected. This procedure is described in Algorithm 2. 
In order to speed up the computation, axis-aligned box $(\mathrm{AAB})$ are used. Each subset $s_{i}$ is associated with an outer $\mathrm{AAB}$ (called also the axis-aligned boundary box) $O A A B\left(s_{i}\right)$ and an inner $\mathrm{AAB}, \mathrm{iAAB}\left(s_{i}\right)$. If $s_{i}$ is defined by the set of $p$ points in $\mathbb{R}^{m+n}\left(\mathbf{x}_{1}, \mathbf{x}_{2}, \ldots, \mathbf{x}_{p}\right)$, then its axis-aligned boundary box $o A A B\left(s_{i}\right)$ is defined as

$$
\begin{aligned}
o A A B\left(s_{i}\right)= & \left\{\mathbf{y} \in \mathbb{R}^{m+n} \mid \forall k, 1 \leq k \leq(m+n),\right. \\
& \left.\min _{1 \leq j \leq l} x_{j}(k) \leq y(k) \leq \max _{1 \leq j \leq l} x_{j}(k)\right\}
\end{aligned}
$$

The inner $A A B$ is an axis-aligned box that is contained within the subset $s_{i}$. As opposed to the outer $A A B$, the definition of the inner $A A B$ is not unique. In this study the inner $A A B$ was centred in the barycentre of the sample points defining $s_{i}$ and its relative size was maximised within $s_{i}$.

The idea behind the inner and outer AABs is that it is extremely cheap to check if a focal element is outside or inside an $\mathrm{AAB}$. The focal elements that are outside the outer $A A B$ are guaranteed not to be within $\mathscr{U}_{v}$ and the one inside the inner $A A B$ are guaranteed to be within $\mathscr{U}_{v}$. Once this selection process is done, only the focal elements that do not enter in any of those categories need to be checked to complete the computation of $\tilde{B e l}$.

Algorithm 2. Cluster approximation method.

Input: $v$, step

$1^{*}$ Fix a low value for threshold $v$

Output: Matrix Out where each row corresponds to a step. The ith row of Out is composed of the value of the threshold, the optimum design vector and the maximum approximated belief found at the $i$ th step.

$X=\{\} ; X_{\text {new }}=\{\}$

$I^{*}$ Initial sample points

$X \leftarrow\{[\mathbf{d}, \mathbf{u}] \mid f(\mathbf{d}, \mathbf{u}) \leq v\}$

$I^{*}$ Initialise $\tilde{B e} l_{\max }$

$\tilde{B e} l_{\text {max }} \leftarrow 0$

while $\tilde{B e l} l_{\max }<1$ do

I*Update the threshold

$v \leftarrow v-$ step

${ }^{*}$ New sampling points

$X_{\text {new }} \leftarrow\{[\mathbf{d}, \mathbf{u}] \mid(v-$ step $)<f(\mathbf{d}, \mathbf{u}) \leq v\}$

$I^{*}$ Update the set of valid sampled point

$X \leftarrow\left\{X, X_{\text {new }}\right\}$

I*Identify the valid subdomains

Partition in clusters the sample points $X$

for each cluster do

Compute the associated convex hull

Compute the oAAB and an $\mathrm{iAAB}$

\section{endfch}

$I^{*}$ Find thedesign point giving the highest $\tilde{B e l} * \mid$

$\left[\tilde{B e l}_{o p t}, \mathbf{d}_{o p t}\right] \leftarrow \max _{\mathbf{d} \in \mathscr{D}} \tilde{\operatorname{Bel}}(\mathbf{d}, v)$

I*Add a line at the end of the output matrix */ and save the results

Out $($ end $+1,:)=\left[v, \tilde{B e l}_{\text {opt }}, \mathbf{d}_{\text {opt }}\right]$

$I^{*}$ Update the optimum belief variable

$\tilde{B e} l_{\max } \leftarrow \tilde{B e} l_{\text {opt }}$

endw
In order to identify if any of the remaining focal elements fulfils the proposition $f(\mathbf{d}, \mathbf{u})<v, \forall \mathbf{u} \in F E$, one only needs to check if their vertices are within any of the subsets $s_{i}$. In our implementation $s_{i}$ is the convex hull of the sample points of the $i$ th cluster. If $\mathbf{v}$ is a point of $\mathbb{R}^{m+n}$, we have

$\mathbf{v} \in s_{i} \Longleftrightarrow \exists \lambda \in\left(\mathbb{R}^{+}\right)^{m+n} \mid \mathbf{v}=\sum_{k=1}^{p} \lambda(k) * \mathbf{x}_{k} \quad$ and $\quad \sum_{k=1}^{p} \lambda(k)=1$

The initial step of the revised simplex method was used to determine whether or not the vector $\lambda$ in (12) exits $[13,14]$.

It is important to highlight that in the cluster approximation, no assumptions are made on the convexity of the system function $f$. Only the subset $s_{i}$ are considered as convex, which in the practical application related to space design appears reasonable. If that was not case, another way to define the subset $s_{i}$ would be required (it could be possible for instance to use an hypersphere or an hypercube of fixed size around each valid sample point). Another advantage of this method is that it shall identify all the locally optimal design regions and thus various classes of interesting designs (as in the direct approach). Finally the global optimum is likely to be found using a simple local optimiser, starting, for instance, from the barycentre of each cluster.

\section{Example of application}

In this section, we will present the results of the three previously described approaches applied to the preliminary design of the BepiColombo mission. The objective is to minimise the wet mass of the spacecraft (for the low-thrust part of the mission) considering uncertainties on a few parameters. The first part of this section presents the calculation of the mass budget of the spacecraft, i.e. the system function $f$.

\subsection{Wet mass modelling}

The mass model in this section was developed for preliminary system mass assessment of a generic Solar Electric Propulsion (SEP) mission. It is mainly based on [15] and allows the estimation of the wet mass of the spacecraft as a function of the thrust profile and specific impulse.

The total SEP related mass is given by the following equation:

$$
\begin{aligned}
m_{\text {wet }}= & m_{\text {tank }}+m_{\text {array }}+m_{\text {rad }}+m_{\text {harness }}+m_{P P U}+m_{\text {thrusters }} \\
& +m_{\text {xenon }}
\end{aligned}
$$

where $m_{\text {tank }}$ is the mass of tanks and associated equipment, $m_{\text {array }}$ is the mass of the solar arrays, $m_{\text {rad }}$ is the radiator and associated structural mass, $m_{\text {harness }}$ is the mass of harness equipment, $m_{P P U}$ is the mass of power processing subsystem, $m_{\text {thrusters }}$ is the mass of the thrusters and structural related mass and $m_{x e n o n}$ is the mass of xenon required to perform the low thrust transfer. The expressions of all these quantities are given in the following subsections. 


\subsubsection{Mass of propellant}

The mass of xenon is estimated from the $\Delta V$ budget using the rocket equation.

$m_{\text {xenon }}=m_{T L O}\left(1-e^{-\Delta V / \overline{I_{S P}} * g_{0}}\right)$

where $m_{T L O}$ is the trans-lunar orbit mass, i.e. the wet mass of the spacecraft just after the escape from the Earth-Moon system (specific to this mission, $m_{T L O}=2400 \mathrm{~kg}$ ), $g_{0}$ is the gravitational acceleration $\left(g_{0}=9.80665 \mathrm{~m} \mathrm{~s}^{-2}\right), \Delta V$ is the delta $V$ budget for the SEP transfer from the Earth-Moon escape to the Mercury capture (in $\mathrm{ms}^{-1}$ ) and $\overline{I_{S P}}$ is the mean specific impulse of the SEP, during transfer (in s):

$\overline{I_{S P}}=\frac{4670}{4720} * I_{\max T}$

In Eq. (15), $I_{S P}$ is the specific impulse at maximum thrust (in s).

4.1.1.1. Delta $V$ budget. The delta $V$ budget is composed of the deep space $\Delta V$ (cf. below), the $\Delta V$ correction for the second Lunar Gravity Assist $\left(40 \mathrm{~m} \mathrm{~s}^{-1}\right)$, the $\Delta V$ penalty for solar aspect angle control $\left(100 \mathrm{~m} \mathrm{~s}^{-1}\right)$, the $\Delta V$ for flyby navigation $\left(260 \mathrm{~m} \mathrm{~s}^{-1}\right)$, the $\Delta V$ for other navigation manoeuvres $\left(280 \mathrm{~m} \mathrm{~s}^{-1}\right)$ and the $\Delta V$ for contingencies ( $+5 \%$ of the deep space $\Delta V)$.

The deep space $\Delta V$ is the total velocity variation, delivered by the SEP system, to transfer the spacecraft from Earth to Mercury. The computation of the deep space $\Delta V$ would require the solution of a computationally expensive optimal control problem for each transfer. On the other hand, all the three approaches to the solution of problem (7) require the evaluation of several design vectors and thus the evaluation of several transfers. Therefore, integrating the solution of the above-mentioned optimal control problem into any of the solution approaches of the OUU would be computationally intractable. In order to overcome this difficulty, a surrogate model [20] was built based on 180 different transfers priorly computed using the EADS-Astrium Stevenage in-house software orbit optimisation facility [15]. Each transfer corresponds to a different value of $P_{1} \mathrm{AU}$, the power generated by the solar arrays at 1 Astronomical Unit (AU), and $T_{\max }$, the maximum thrust. For this study, the DACE package [16] was used to generate a Kriging surrogate model with a first order polynomial regression model and an exponential correlation model (cf. Fig. 5).

\subsubsection{Tank mass}

The mass of the tanks is estimated with:

$m_{\text {tank }}=\sigma_{\text {tank }} * m_{\text {xenon }}$

where $\sigma_{\text {tank }}$ is the specific ratio of the tank subsystem $\left(\sigma_{\operatorname{tank}}=\right.$ $11 \%)$.

\subsubsection{Solar arrays}

For the solar arrays we need both the mass and the area.

4.1.3.1. Array area. The area of the solar arrays is a function of the power required at $1 \mathrm{AU}$ :

$A=\frac{P_{1 \mathrm{AU}}}{\eta_{p} * G_{S}} * \kappa_{A}$

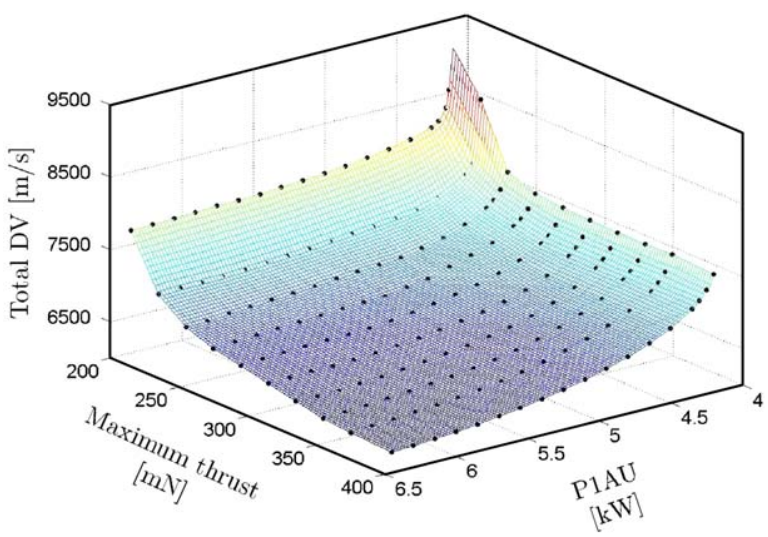

Fig. 5. Kriging surrogate of the deep space $\Delta V$ for the BepiColombo low thrust transfers.

where $\eta_{p}$ is the power conversion efficiency $\left(\eta_{p}=0.22751\right)$, $G_{S}$ is the solar constant at $1 \mathrm{AU}\left(G_{S}=1367 \mathrm{Wm}^{-2}\right)$ and $\kappa_{A}$ is the area margin for the solar arrays $\left(\kappa_{A}=1.2\right)$.

4.1.3.2. Array mass. The mass of the solar arrays is then derived from their area with:

$m_{\text {array }}=\left(A * \rho_{S A}+m_{\text {array }}\right) * \kappa_{S A}$

where $\rho_{S A}$ is the specific ratio mass/area of the solar arrays $\left(\rho_{S A}=2.89 \mathrm{~kg} \mathrm{~m}^{-2}\right), m_{0}$ is the inevitable structural mass of the solar arrays and $\kappa_{S A}$ is the mass margin for the solar arrays $\left(\kappa_{S A}=1.1\right)$.

\subsubsection{Radiator and associated structural mass}

The radiator is sized based on the maximum power delivered at the shortest distance from the Sun. In the case of the BepiColombo mission, this is at the perihelion of Mercury's orbit, i.e. $0.3 \mathrm{AU}$.

4.1.4.1. Maximum power. $P_{\max }$ is the total power used by the thrusters at maximum thrust, i.e. at $0.3 \mathrm{AU}$. It is calculated using a system of two equations linking the power used by the thrusters, the thrust, the specific impulse and the voltage. The power is a linear function of the thrust while the specific impulse is a second order polynomial of the thrust:

$I_{S P}=b_{2} T^{2}+b_{1} T+b_{0}$

$P=c *\left(a_{1} T+a_{0}\right)$

where $a_{1}, a_{0}, b_{2}, b_{1}$ and $b_{0}$ linear functions of $\sqrt{V}$ ( $V$ is the voltage in volt and $c$ a constant). The voltage is first computed using Eq. (19) and then $P_{\max }$ is calculated using Eq. (20) with $T=T_{\max }$ and $I_{S P}=I_{\max T}$.

4.1.4.2. Dissipated power. The total power that needs to be dissipated through the radiator is given by

$P_{\text {dis }}=\delta_{p} P_{\max }+Q$

where $\delta_{p}$ is the percentage of the maximal power that is wasted ( $\left.\delta_{p}=0.15\right), Q$ is the heat to be dissipated at the 
perihelion of Mercury's orbit and $P_{\max }$ is the power used by the thrusters at $T_{\max }$ and $I_{S P}$, i.e. at $0.3 \mathrm{AU}$.

4.1.4.3. Radiator and associated structural mass. Two different types of radiator were envisaged for BepiColombo. The choice depends on the value of the dissipated power in Eq. (21) being above or below a given threshold $P_{\text {dim }}$. The mass of the radiator and of the associated structure is calculated using the following equation:

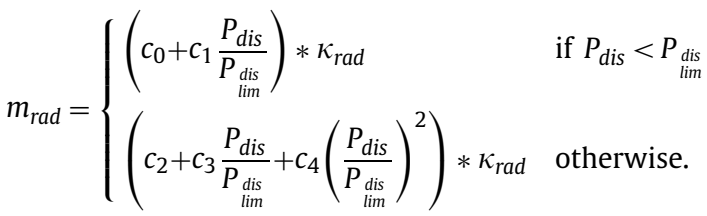

where $c_{0}, c_{1}, c_{2}, c_{3}$ and $c_{4}$ are constants and $\kappa_{\text {rad }}$ is the mass margin for the radiator $\left(\kappa_{\text {rad }}=1.15\right)$.

\subsubsection{Harness}

The mass of the harness is given by the following equation:

$m_{\text {harness }}=m_{\text {harness }}+\rho_{\text {harness }} P_{\max } \kappa_{\text {harness }}$

where $m_{\text {harness }}$ is the inevitable mass of the harness subsystem, $\rho_{\text {harness }}$ is the specific ratio mass/power of the harness subsystem $\left(\rho_{\text {harness }}=1.3763 \times 10^{-3} \mathrm{~kg} \mathrm{~W}^{-1}\right)$ and $\kappa_{\text {harness }}$ is the mass margin for the harness subsystem $\left(\kappa_{\text {harness }}=1.2\right)$.

\subsubsection{Power processing unit}

BepiColombo was designed with four power processing unit (PPU). The mass of each of them is estimated using an equation linear with the maximum power $P_{\max }$ and quadratic with the mean specific impulse $\overline{I_{S P}}$.

\subsubsection{Thrusters mass}

The mass of the thrusters was estimated with the following equation:

$m_{\text {thrusters }}=m_{\text {thrusters }}^{0}+n_{\text {thruster }} m_{\text {nominal }}^{\text {nhrusters }}$

where $m_{\text {thrusters }}$ is the inevitable mass of the thrusters sub-

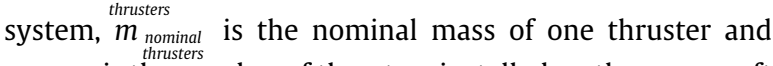
$n_{\text {thruster }}$ is the number of thrusters installed on the spacecraft $\left(n_{\text {thruster }}=2\right)$.

\subsubsection{Remarks}

The simple model presented here enables the estimation of the mass of the main subsystems of a low thrust spacecraft with only three design parameters:

- $P_{1 \mathrm{AU}}$ : the power to be generated by the solar arrays at $1 \mathrm{AU}$.

- $T_{\max }$ : the maximum thrust.

- $I_{S P}:$ the specific impulse at maximum thrust.

If no uncertainties in the model are considered, an optimal design point would correspond to a triplet of values, assigned to $P_{1 \mathrm{AU}}, T_{\max }$ and $I_{\max T}$, which minimises the
Table 1

Margins applied to the mass budget of BepiColombo.

\begin{tabular}{lll}
\hline Margins & Value & Subsystem \\
\hline$\Delta V$ & $+5 \%$ & $\Delta V$ Contingency \\
$\kappa_{A}$ & 1.20 & Area of the solar arrays \\
$\kappa_{S A}$ & 1.10 & Mass of the solar arrays \\
$\kappa_{\text {rad }}$ & 1.15 & Mass of the radiator \\
$\kappa_{\text {harness }}$ & 1.20 & Mass of the harness subsystem \\
\hline
\end{tabular}

Table 2

Uncertainty representation through Evidence Theory.

\begin{tabular}{llll}
\hline $\begin{array}{l}\text { Uncertain } \\
\text { parameter }\end{array}$ & Intervals & $\begin{array}{l}\text { Basic probability } \\
\text { assignment }\end{array}$ \\
\cline { 2 - 3 } & Lower bound & Upper bound & \\
\hline$\eta_{p}$ & 0.18959 & 0.195 & 0.05 \\
& 0.195 & 0.205 & 0.15 \\
& 0.205 & 0.215 & 0.25 \\
& 0.215 & 0.22751 & 0.55 \\
$\rho_{S A}$ & 2.89 & 3.00 & 0.10 \\
& 3.00 & 3.10 & 0.15 \\
& 3.10 & 3.25 & 0.35 \\
& 3.25 & 3.3105 & 0.40 \\
$\rho_{\text {harness }}$ & $1.3763 \times 10^{-3}$ & $1.4500 \times 10^{-3}$ & 0.05 \\
& $1.4500 \times 10^{-3}$ & $1.5500 \times 10^{-3}$ & 0.25 \\
& $1.5500 \times 10^{-3}$ & $1.6000 \times 10^{-3}$ & 0.30 \\
& $1.6000 \times 10^{-3}$ & $1.6515 \times 10^{-3}$ & 0.40 \\
\hline
\end{tabular}

mass budget. However, although optimal, the resulting design point would not be reliable as the mass of system is known to increase, from the preliminary design to launch, for the effect of the initial uncertainties. If a margin approach is used to predict the mass budget, it is common practise to increase the mass of poorly known components by 20-25\%, the mass of known components by $10-15 \%$ and the mass of consolidated designs by $5-10 \%$ [21]. Note that, these percentages are not derived from a rigorous calculation of the propagation of uncertainties through the design process, but are based on historical data and past experience. Furthermore, there is no prediction of any event, during the development of the design process, that can further increase the mass beyond the prescribed margins.

In the case of BepiColombo, margins were added to the mass of the propellant (by an increase of the $\Delta V$ budget), to the mass and area of the solar arrays, to the mass of the radiator and to the mass of the harness (see Table 1 ). Since the estimation of the mass of these components was deemed to be uncertain, in the following, we will use Evidence Theory to model the related uncertainty and to study its propagation throughout the system model.

\subsection{The BPA structure}

We assume here that the major source of uncertainty comes from the power conversion efficiency $\eta_{p}$, the area density of the solar array $\rho_{S A}$ and the mass/power ratio of the harness $\rho_{\text {harness }}$. Note that, in a real scenario, this choice is dependent on the technology readiness of the components, the current stage of the design, the expert opinion of the designers. Therefore, more or different parameters can be deemed to be uncertain. Table 2 presents the BPA structure associated to the parameters selected for this study. 
Since for the OUU problem, Evidence Theory is used to model the uncertainties related to $\eta_{p}, \rho_{S A}$ and $\rho_{\text {harness }}$, their margins, i.e. $\kappa_{A}, \kappa_{S A}$ and $\kappa_{\text {harness }}$, were set to 1 .

In order to easily compare margin approach and robust design based on Evidence Theory[ ], the BPA structure was constructed in such a way that the uncertainty interval of a given parameter ranges from the nominal value of the parameter to its nominal value times the corresponding margin.

\subsection{Problem formulation}

Using the notation of Section 2.3, let us define $\mathbf{d}$, $\mathbf{u}$ and their associated domains $\mathscr{D}$ and $\mathscr{U}$, as

$$
\begin{aligned}
\mathbf{d}= & {\left[P_{1 \mathrm{AU}}, T_{\max }, I_{\max T}\right] } \\
\mathscr{D}= & {[4200 \mathrm{~W}, 6450 \mathrm{~W}] \times[210 \mathrm{mN}, 400 \mathrm{mN}] } \\
& \times[4000 \mathrm{~s}, 8000 \mathrm{~s}] \\
\mathbf{u}= & {\left[\eta_{p}, \rho_{S A}, \rho_{\text {harness }}\right] } \\
\mathscr{U}= & {[0.18959,0.22751] \times[2.89,3.3105] } \\
& \times\left[1.3763 \times 10^{-3}, 1.6515 \times 10^{-3}\right]
\end{aligned}
$$

The OUU, then, becomes:

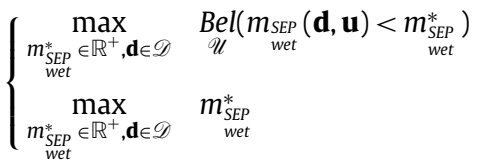

\section{Deterministic optimal design}

For the case under investigation, the identification of an optimal design point, when the margin approach is applied, requires the solution of the following minimisation problem:

$\min _{\mathbf{d} \in \mathscr{D}} m_{\text {SEP }}(\mathbf{d})$

where, margins $\kappa_{A}, \kappa_{S A}$ and $\kappa_{\text {harness }}$ in Table 1 are applied to the corresponding parameters $\eta_{p}, \rho_{S A}$ and $\rho_{\text {harness. }}$. Here and in the following, we call (30) deterministic optimal design problem. The deterministic optimal design problem aims at finding the minimum wet mass of the spacecraft by adjusting the design vector $\mathbf{b}$, given a predefined value of $\eta_{p}, \rho_{S A}$ and $\rho_{\text {harness }}$. The best-known solution to problem (30), found using a global optimiser, is $m_{\text {SEP }}=883.880 \mathrm{~kg}$ which corresponds to $\mathbf{d}_{d e t}=[4650.0 \mathrm{~W}, 230.00 \mathrm{mN}, 5648.0 \mathrm{~s}]$. For this design vector, the mass without the three margins considered in this test case ( $\kappa_{A}, \kappa_{S A}$ and $\left.\kappa_{\text {harness }}\right)$ would be $864.8 \mathrm{~kg}$. Therefore, it can be said that the margin approach predicts and increase of the system mass by $19 \mathrm{~kg}$. However, the the solution of problem (30) does not yield any measure of the reliability of the deterministic design vector $\mathbf{d}_{\text {det }}$ and no information on the trade-off between reliability and performance

Although this is not generally the case, for the sake of the comparison between margin approach and robust design optimisation based on ET, we assumed that the vector $\mathbf{d}_{\text {det }}$ corresponds to the best design with maximum reliability.

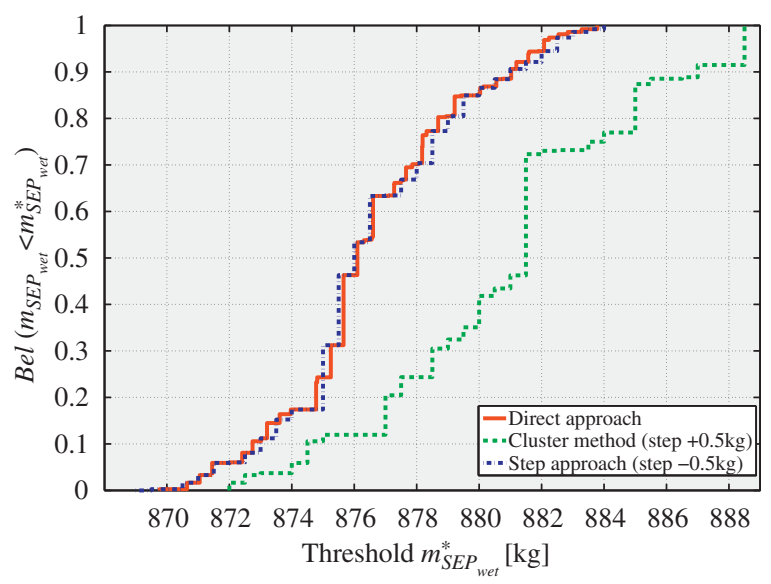

Fig. 6. Comparison of the results given by the three proposed methods (direct, step and cluster) to solve the OUU problem for the BepiColombo test case.

Because of this assumption, together with the choice of the BPA structure, the optimal design of the OUU for a belief of 1, i.e. full certainty, is expected to be the same as the deterministic one. This is obviously not generally the case but will help here to better comprehend the results. i.e. it was expected that the OUU yielded a Belief equal to 1 for $\mathbf{d}=\mathbf{d}_{\text {det }}$

\section{Comparison of the three proposed methods}

\subsection{General results}

For the step method, the initial threshold was set to $920 \mathrm{~kg}$ and then decreased by $0.5 \mathrm{~kg}$ at each iteration. The initial $\mathbf{d}$ chosen was the centre of the domain $\mathscr{D}$. A total of 72 iterations were required to find the minimum mass corresponding to a Belief equal to 1 , and 30 more to find the mass for which the Belief is equal to 0 for any design vector. Note that the first 72 iterations are very quick as the optimisation was stopped as soon as a belief equal to 1 was found. The step method yielded a minimum mass with maximum reliability (Belief =1) $m_{\substack{S E P \\ \text { wet }}}^{*}=884.0 \mathrm{~kg}$.

For the cluster approximation, the initial threshold was set to $872 \mathrm{~kg}$ and it was increased by $0.5 \mathrm{~kg}$ at each iteration. A total of 34 iterations where required to reach a Belief of 1 , corresponding to a minimum mass with maximum reliability $($ Belief $=1) m_{\substack{S E P \\ \text { wet }}}^{*}=888.5 \mathrm{~kg}$.

Finally, for the direct method using the MOO, the threshold $m_{S E P}^{*}$ was bound within the interval [ $\left.860 \mathrm{~kg}, 900 \mathrm{~kg}\right]$. After about 100,000 evaluation of the Belief, 53 different Pareto optimal points were found, all in the same neighbourhood. Once again, the minimum mass with maximum reliability $($ Belief $=1)$ was $m_{\substack{\text { SEP } \\ \text { wet }}}^{*}=883.9 \mathrm{~kg}$.

\subsection{Trade-off curves and pareto fronts}

Fig. 6 shows the Pareto fronts identified by the three proposed method. It can be seen that the Direct Approach and 
Step Method give the best results. They both found the optimum design for a belief of 1 . As expected, the optimum design for a Belief of 1 is the same as the deterministic optimum. The cluster approximation gives the worst Pareto front but still remains quite accurate $(<10 \%$ of increase of the mass). Moreover, the result is conservative (higher threshold for the same Belief level) thus more robust.

Compared to the solution of problem (30), the curves in Fig. 6 provide two additional pieces of information: a quantitative measure of the belief that the predicted wet mass will be lower than the threshold, given the current information; and the exact measure of the relationship between reliability and performance, given the current information. The latter aspect allows a quantitative trade-off analysis between reliability and performance, giving a full range of design options. It is important to underline that the result in Fig. 6 is only based on the available information at the time of the solution of the OUU and cannot be used to predict the future occurrence of events, during the design process, that will introduce further perturbations to the value of the wet mass.

\subsection{Localisation of optimal designs}

A second important outcome of these tests is related to the localisation of the optimum design found by the three approaches. As already mentioned in Section 2.3, the designer is interested in identifying different design options that may be optimal at different level of belief.

The step method, after finding the deterministic optimum for a Belief of 1, converged, for all the subsequent levels of Belief, to the same design point $[4801.3 \mathrm{~W}, 230.02 \mathrm{mN}$, $5648.8 \mathrm{~s}$ ].

On the other hand, the Direct approach identified mainly three different design options. For a threshold above $880 \mathrm{~kg}$ all the solutions are in a neighbourhood of the deterministic optimum, for a threshold between 880 and $877 \mathrm{~kg}$ all the solutions are around the design vector [ $4760 \mathrm{~W}, 230 \mathrm{mN}, 5650 \mathrm{~s}]$ and finally, for the rest of the curve, the Direct approach converges to the two previous design options along with a third one in the neighbourhood of [ $4815 \mathrm{~W}, 230 \mathrm{mN}, 5629 \mathrm{~s}]$. Fig. 7 shows the power at $1 \mathrm{AU}$ of the optimum design points found for various belief levels.

The cluster method did identify only one design option in the vicinity of the solution vector [ $5250 \mathrm{~W}, 235 \mathrm{mN}, 5650 \mathrm{~s}]$. This design option is not optimal for any level of belief as the wet mass is not minimal. The Pareto optimal design solutions identified by the Direct approach were within the feasible domains enclosed by the convex hulls. However, they generate approximated beliefs $\tilde{B e l}$ that are not maximal. This problem could be due to the sampling technique or the use of convex hull, and further investigations and tests are require to overcome this drawback.

\subsection{Computational cost}

As shown in the previous section, the three solution methods display substantially different behaviours and can lead to quite different results in terms of number and quality of the solutions. Furthermore, their computational cost

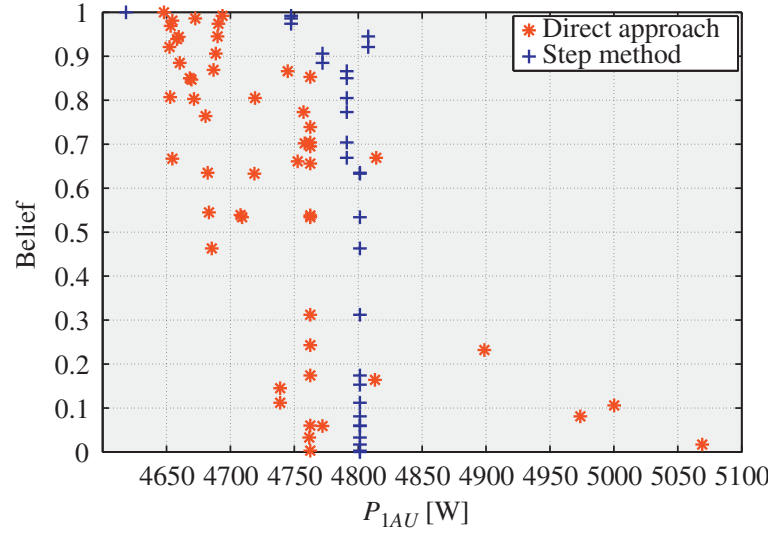

Fig. 7. Localisation of the optimum power at $1 \mathrm{AU}$ found vs. the belief level, found by the direct approach and the step method for the BepiColombo test case.

Table 3

Computational time for the three proposed approaches.

\begin{tabular}{lccc}
\hline Method & $\begin{array}{l}\text { Total CPU } \\
\text { time }(\mathbf{s})\end{array}$ & $\begin{array}{l}\text { CPU time } \\
\text { for } \boldsymbol{f}(\mathbf{s})\end{array}$ & $\begin{array}{l}\text { Number of } \boldsymbol{f} \\
\text { evaluations }\end{array}$ \\
\hline Step & 288 & 133 & 330,375 \\
Cluster & 1206 & 59 & 135,803 \\
Direct & 3859 & 1926 & $7,122,580$ \\
\hline
\end{tabular}

is substantially different. Table 3 summarised the computational time of each method applied to the solution of the OUU of BepiColombo.

The step approach takes 13 times less than the Direct approach, yields a similar Pareto front but provides only 1 design option. The cluster approximation requires about 3 times less function evaluations of the step approach and 52 times less function evaluations than the Direct approach but produces a worse Pareto front. As the system function can be computationally expensive, a lower number of call to $f$ is particularly attractive making the cluster approximation quite interesting. To give an idea, in our case, a system function evaluation requires around $4 \times 10^{-4}$ seconds. If instead the CPU time for computing the wet mass of the SEP system was over $5 \times 10^{-3}$, the cluster approximation would become faster than the step approach.

The higher cost of the cluster method is mainly due to the generation of the convex hull. A more efficient way of dealing with the identification of $S_{v}$ is under development and will be the subject of a future publication.

The high number of function evaluations associated to the Direct approach, instead, is mainly due to the nature of the dominance index (as explained in Section 3.1) and to the identification of multiple design options. This later aspect, in particular, requires the exploration of multiple neighbourhoods with a consequent increase in the number of function evaluations.

\subsubsection{Influence of the number of focal elements}

The same simulations was run with only the cluster approximation and the step method for an increasing number of focal elements (i.e. the number of intervals per in certain 


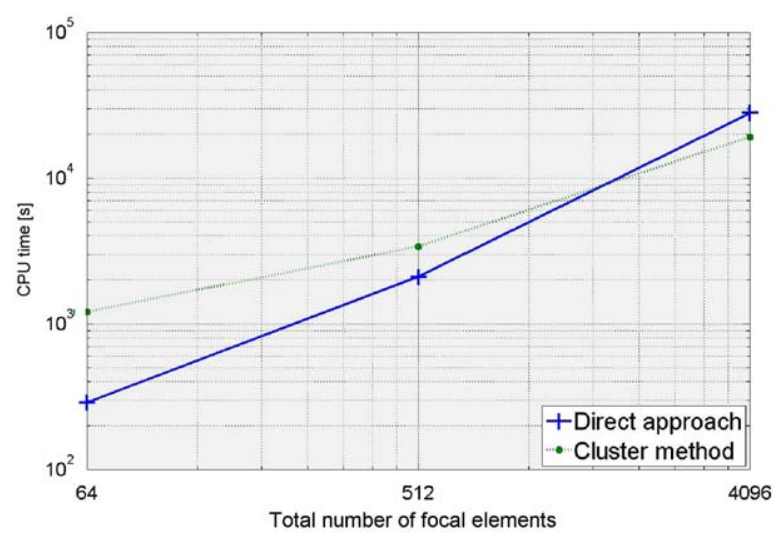

Fig. 8. Variation of the computational time for the cluster approximation and the step method for increasing number of focal elements.

parameters has been increased to 8 and 16). Fig. 8 shows how the cluster approximation can become even more attractive, in terms of computational time, when building the focal elements is expensive.

\section{Conclusion and future work}

In this paper, we have presented an initial investigation of the use of robust design optimisation, based on Evidence Theory[ ], to deal with uncertainties inherent to the preliminary design of a space mission. Evidence Theory appeared to be an appropriate choice as, in this phase, epistemic uncertainties are frequently encountered. The main benefits that can be derived from this initial investigation are: (a) system margins are replaced by a more meaningful and rigorous uncertainty quantification, directly related to the source of uncertainties and to the current level of information, (b) multiple design options can be identified, corresponding to different levels of performance and reliability, (c) the decision maker is presented with a complete quantitative trade-off between performance and reliability and (d) the iteration process between optimisation and reliability analysis is reduced if not avoided.

Three different approaches to solve the optimisation under uncertainties problem were proposed and tested on a realistic preliminary space mission design problem. Each of them had some pros and cons. The step method appeared efficient for problems with convex and inexpensive system functions. However, it does not guarantee the identification of the global optimum for each level of the Belief. The cluster approximation produces acceptable results and appears to be very attractive for non-convex, multi-modal and expensive system functions. Finally, the use of an MOO removes the issue of selecting the steps between two successive thresholds and most importantly identify different optimal design classes, but at the expense of a higher computational time.

As highlighted in the paper, future work is necessary mainly on the cluster approximation. The quality of the sample is of high importance for good results, and more investigation is required here. Moreover, other method could replace the use of convex hull to characterise the valid domains. Regarding the direct approach, a redefinition of the local Pareto optimality criterion would be needed to reduce the computational time and will be presented in a future work. Additionally, other test cases with more uncertain parameters and with more than one local minimum are required to complete the comparison among different approaches.

\section{Acknowledgement}

The BepiColombo model used in this study was kindly provided by EADS-Astrium Stevenage. We greatly appreciated their help and support.

\section{References}

[1] A.P. Dempster, Upper and lower probabilities induced by a multivalued mapping, Ann. Math. Stat. 38 (1967)

[2] G. Shafer, A Mathematical Theory of Evidence, Princeton University Press, Princeton, 1976.

[3] W.L. Oberkampf, J.C. Helton, Investigation of evidence theory for engineering applications, in: Fourth Non-Deterministic Approaches Forum, vol. 1569, AIAA, April 2002.

[4] M. Vasile, Robustness optimisation of aerocapture trajectory design using a hybrid co-evolutionary approach, in: 18th International Symposium on Space Flight Dynamics, vol. 548, ESA Special Publication, 2004.

[5] M. Vasile, D. Bonetti, Evolution of the concurrent design process under uncertainties, in: International Concurrent Engineering Workshop, ESA, 2004.

[6] J.C. Helton, Uncertainty and sensitivity analysis in the presence of stochastic and subjective uncertainty, J. Stat. Comput. Simul. 57 (1997).

[7] K. Sentz, S. Ferson, Combination of evidence in Dempster-Shafer theory, in: Sixth World Multi-conference on Systemics, Cybernetics and Informatics, July 2002.

[8] H. Agarwal, E. John Renaud, L. Evan Preston, Trust region managed reliability based design optimization using evidence theory, Collection of Technical Papers-AIAA/ASME/ASCE/AHS/ASC Structures Structural Dynamics and Materials Conference 5 (2003) 3449-3463.

[9] H.-R. Bae, V. Ramana Grandhi, A. Robert Canfield, Uncertainty quantification of structural response using evidence theory, Collection of Technical Papers-AIAA/ASME/ASCE/AHS/ASC Structures Structural Dynamics and Materials Conference 4 (2002) 2135-2145.

[10] M. Vasile, Robust mission design through evidence theory and multiagent collaborative search, Ann. N Y Acad. Sci. 1065 (2005) 152-173.

[11] M. Vasile, Hybrid Behavioural-Based Multiobjective Space Trajectory Optimization, on Multi-Objective Memetic Algorithms, in: C.-K. Goh, Y.-S. Ong, K.C. Tan (Eds.), Springer Series of Studies in Computational Intelligence, Series: Studies in Computational Intelligence, vol. 171, XII, 2009, 404pp., 227 illus., Hardcover, ISBN: 978-3-540-88050-9.

[12] C. Maddock, M. Vasile, Design of optimal spacecraft-asteroid formations through a hybrid global optimization approach, Int. J. Intell. Comput. Cybernet. 1 (2) (2008) 239-268.

[13] G.B. Dantzig, Linear Programming and Extensions, Princeton University Press, Princeton, 1965.

[14] B.D. Bunday, Basic Linear Programming, Hodder Arnold, 1984.

[15] S. Kemble, Interplanetary Mission Analysis and Design, Springer Praxis Books, 2006.

[16] S.N. Lophaven, H.B. Nielsen, J. Sondergaard, DACE: a MatLab kriging toolbox, Technical Report IMM-TR-2002-12, Technical University of Denmark, 2002.

[17] H.-G. Beyer, B. Sendhoff, Robust optimization: a comprehensive survey, Comput. Methods Appl. Mech. Eng. 196 (2007) 3190-3218.

[18] X. Du, Y. Wang, W. Chen, Methods for robust multidisciplinary design, 41st AIAA/ASME/ASCE/AHS/ASC Structures, Structural Dynamics and Materials Conference and Exhibit 1785 (2000).

[19] H. Agarwal, J.E. Renaud, E.L. Preston, D. Padmanabhan, Uncertainty quantification using evidence theory in multidisciplinary design optimization, Reliab. Eng. Syst. Safety 85 (1-3) (2004) 281-294.

[20] D.R. Jones, A taxonomy of global optimization methods based on response surfaces, J. Global Optim. 21 (2001) 345-383.

[21] R. James Wertz, J. Wiley Larson, Space Mission Analysis and Design, third ed., Kluwer Academic Press, Dordrecht, 1999. 